\title{
DIEZ AÑOS DE SERVICIO DE APOYO A LA FORMACIÓN INTEGRAL DEL ESTUDIANTE EN EL DEPARTAMENTO DE FÍSICA: ASESORÍA ENTRE PARES
}

EPISTEMUS

ISSN: 2007-8196 (electrónico)

ISSN: 2007-4530 (impresa)

\section{Ignacio Cruz Encinas 1}

Francisco Nemesio Armenta Aguilar 2

Laura Lorenia Yeomans Reyna ${ }^{3}$

Denniz Márquez Ruíz 4

Roberto Pedro Duarte Zamorano 5

Recibido: 15 de marzo de 2018,

Aceptado: 26 de mayo de 2018

Autor de Correspondencia:

MIE. Ignacio Cruz Encinas

Correo: ignacio.cruz@fisica.uson.mx

\section{Resumen}

Los asesores pares son estudiantes de los últimos semestres con alto desempeño académico que comparten sus conocimientos, experiencia y facilitan la integración al ámbito universitario de los estudiantes de los primeros semestres. Enriquecido con la experiencia de 10 años de servicio ininterrumpido y las actuales disposiciones administrativas, se presenta armonizado el nuevo protocolo del proyecto académico de docencia de asesores pares. Se parte del principio filosófico de que es un proyecto de apoyo a los estudiantes, es decir, se justifica por sí mismo, por su propia naturaleza por la cual fue concebido, es "per se". No pretende alcanzar, mantener o rebasar indicadores institucionales de calidad tales como: promedio de calificaciones, índice de aprobación, tasa de rendimiento por materia, cantidad de asesorías, alumnos atendidos, etc. Aún así, se ha encontrando que más del $80 \%$ de los alumnos que acuden a asesoría de pares acreditan la asignatura y, quiénes lo hacen, con un promedio de calificación por arriba de la media institucional.

Palabras clave: Asesoría académica, pares, reprobación, aprovechamiento.

\section{Abstract}

Peer advisors are students of the last semesters with high academic performance who share their knowledge, experience and facilitate the integration into the university environment of the students of the first semesters. Enriched with the experience of 10 years of uninterrupted service and the current administrative provisions, the new protocol of the academic project of teaching of peer advisers is presented harmonized. It is based on the philosophical principle that it is a project of support to students, that is, it justifies itself, by its very nature for which it was conceived, is "per se". It does not aim to achieve, maintain or exceed institutional quality indicators such as: average grades, passing rate, subject matter rate, number of consultants, students attended, etc. Even so, it has been found that more than $80 \%$ of the students that go to peer counseling accredit the subject and, who do, with an average grade above the institutional average.

Keywords: Academic advisory, peers, reprobation, academic performance. DEPARTAMENTO DE FISICA / UNIVERSIDAD DE SONORA, MÉXICO / laura.yeomans@fisica.uson.mx 3 DEPARTAMENTO DE FISICA / UNIVERSIDAD DE SONORA, MÉXICO / denniz.marquez@fisica.uson.mx 4 DEPARTAMENTO DE FÍSICA / UNIVERSIDAD DE SONORA, MÉXICO / roberto.duarte@fisica.uson.mx 5 


\section{INTRODUCCIÓN}

La deserción en el nivel superior es un fenómeno multidimensional y complejo en donde se conjugan factores socioculturales, políticos, psicológicos, pedagógicos e institucionales [1]. Para los alumnos, los primeros semestres y los primeros meses en el nivel superior, pueden ser definitivas para el logro de los objetivos y metas educativas ya que tienen que desarrollar habilidades para su integración a la comunidad universitaria y afrontar problemas derivados de las nuevas relaciones sociales. Para facilitar la integración social es necesario crear ambientes y condiciones propicias que permitan el intercambio de experiencias entre los jóvenes que ingresan y los que ya han adquirido su identidad institucional.

De acuerdo al ámbito, existen teorías y modelos que buscan explicar la deserción escolar. Uno de ellos, el modelo de la organización, intenta explicar el fenómeno a partir de la complejidad institucional, sus recursos y los ambientes de aprendizaje.

Otro modelo, el de interacción social, lo hace a partir de la socialización entre los diferentes actores del proceso educativo asumiendo que la conducta es el resultado de la interacción reciproca entre los ambientes y los individuos y entre ellos mismos. Tiene su referente teórico en la teoría sociocultural de Vigotsky [2], que hace referencia a la reducción de la distancia entre la zona de desarrollo real (ZDR) determinada por la capacidad de resolver de forma independiente un problema, y la zona de desarrollo potencial (ZDP) determinada a través de la resolución de un problema bajo la guía de un experto o en colaboración con un compañero más capaz. Según esta teoría constructivista, los procesos psicológicos de orden superior se adquieren primero en un contexto social mediado por el lenguaje $y$, si son significativos, se interiorizan, es decir, las funciones se adquieren primero en escala social (interpsicológica) y posteriormente en lo individual (intrapsicológica), alcanzando el aprendiz el desarrollo cognitivo producto de su socialización con el medio.

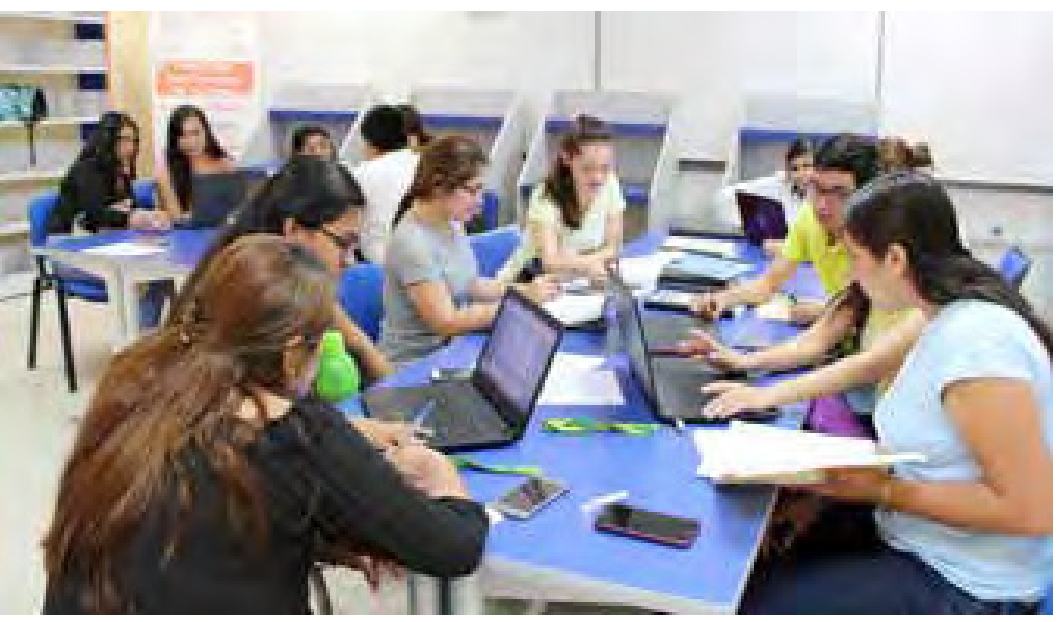

Para facilitar la integración social es necesario crear ambientes y condiciones propicias que permitan el intercambio de experiencias recientes entre los jóvenes, situación que, en algunos casos, resulta difícil de establecer entre un maestro o tutor y el alumno, debido a la figura de autoridad que tiene el primero y la personalidad y características individuales del segundo, según dan testimonio los mismos alumnos al mencionar: "algunos estudiantes en riesgo no se acercan a los maestros por algún motivo (vergüenza u de otro tipo) y suponemos que puede existir un lazo de confianza mayor entre estudiantes" [3].

En estos primeros semestres prevalece la deserción, que Tinto [4] atribuye al desconocimiento de la Institución e insuficiente integración personal con el ambiente intelectual y social, o por que la Institución no les brinda apoyo extra en asignaturas que se les dificultan comprender, entre las cuales se encuentran las ciencias básicas como la Física y Matemáticas. De acuerdo con este autor, los primeros dos factores representan el primer periodo crítico para los alumnos de nuevo ingreso, en tanto que la falta de apoyos institucionales representa su segundo periodo crítico.

Se comparte la idea que a nivel nacional han venido gestando y compartiendo experiencias diferentes tutores: Jiménez y Torres [5]; Castro y López [6]; Alamilla y Puga [7]; Burgos, Couoh y Quintana [8]; Cantú, Siller y García [9] de que, con la incorporación de alumnos pares de alto rendimiento académico y de semestres avanzados que brinden asesorías a alumnos de los primeros semestres en aspectos académicos e institucionales, las partes involucradas tendrán la posibilidad de adquirir conocimiento en un ambiente más relajado, con un mayor nivel de confianza e identificación, a la vez que los últimos contaran con un asesor más próximo en edad que el profesor más joven que pueda tener la institución, siendo los asesores pares quienes mejor conocen las dificultades a las que ellos mismos se enfrentaron y pueden tener mayor capacidad para detectar problemas de convivencia, así como quienes mejor pueden implementar estrategias para mejorar la convivencia, las relaciones interpersonales y contribuir a disminuir los índices de reprobación y deserción.

\section{OBJETIVO GENERAL DEL PROYECTO}

Instrumentar una estrategia de enlace entre los estudiantes de nuevo ingreso y los que cursan sus últimos semestres, con la intención de reducir los casos de reprobación y deserción entre alumnos por insuficiente integración social e incrementar el aprovechamiento y la eficiencia terminal, creando ambientes propicios que favorecen el aprendizaje significativo y la formación integral, a partir de la comunicación y el intercambio de experiencias entre pares, con un mayor nivel de confianza e identificación entre iguales. 
Generar una instancia a donde los alumnos tutorados, detectados como vulnerables, con bajo rendimiento académico o en riesgo escolar en asignaturas de Física y Matemáticas del Eje de Formación Común, puedan ser canalizados por sus tutores o Coordinadores de Programas Educativos cuyos planes de estudio contengan dichas asignaturas.

\section{JUSTIFICACIÓN INSTITUCIONAL}

\section{Tasa de retención}

En la Universidad de Sonora, de acuerdo con estudios de deserción de cohortes de alumnos que ingresaron a la Universidad de Sonora en los años 2005 [10], 2007 [11] y 2009 [12], realizados por la Dirección de Planeación, la mayor deserción a nivel institucional se presenta en el primer año de la carrera.

Después de un año de haber ingresado la generación de alumnos del 2005, el indicador de deserción institucional se ubicaba en $12.8 \%$. En el segundo estudio de deserción de la generación 2007, al siguiente año dejó de reinscribirse el $24.6 \%$ de los alumnos. Resultados del tercer estudio de deserción practicado a la generación 2009 , indica que al tercer semestre ya no se reinscribió el 19.6\%.

Entre las causas destacan: abandono de estudios, incumplimiento de requisitos de ingreso, bajas voluntarias, suspensión de alumnos del tronco común al no alcanzar un rendimiento académico suficiente y bajas por reglamento escolar.

En los tres estudios de deserción (2005, 2007 y 2009), la División de Ciencias Exactas y Naturales es la que presenta menor porcentaje de ingreso. En contraparte, presenta la mayor deserción para reinscripción al segundo año (la generación 2005 presentó un 19.4\%; la cohorte del 2007 la deserción se ubicó en un $27.6 \%$, en tanto que para la generación 2009 se reporta un porcentaje de permanencia del $64.4 \%$, es decir, una deserción del $35.6 \%$ ). En estas tres generaciones de alumnos, la Licenciatura en Matemáticas y Ciencias de la Computación, se reportan como dos de los cinco programas de la DCEN con índices de retención menores al $60 \%$.

La División de Ciencias Químico Biológicas es la segunda División con mayor índice de deserción, donde los índices de retención para los mismos años en que se hicieron los estudios son: $87.7 \% ; 73 \%$ y $82 \%$ para las generaciones 2005, 2007 y 2009 respectivamente.

En la División de Ingenierías, después de un año de haber ingresado la generación de alumnos del 2005 en alguna de sus Licenciaturas, el estudio indica que el $15.9 \%$ ya no se reinscribió en el segundo semestre del 2006; para la generación 2007 la deserción fue del 25.4\%, en tanto que para la 2009, este mismo indicador se ubico en un $27 \%$.

\section{Índice de reprobación por División}

En la sección de Sistema de Información Estadística
(SIE) de la página electrónica de la Dirección de Planeación [13], se publican los índices de reprobación por materia por División y por programa educativo, el cual resulta del cociente del total de materias reprobadas entre el número total de materias cursadas en un semestre por el conjunto de alumnos, multiplicado por cien. A partir de la información que comprende del semestre 2004-1 al 2007-1, el índice de reprobación para la Unidad Regional Centro era del $14.51 \%$, con datos actualizados al semestre 2016-2, la institución muestra un avance, ya que ha logrado reducir este indicador a un 12.37\%. La División de Ciencias Exactas y Naturales presenta en ambos periodos (2004-1 a 2007-1 y 2004-1 a 2016-2) el más alto índice de reprobación institucional ( $25.59 \%$ en el primero y $21.08 \%$ en la actualidad), el segundo lugar lo ocupa la División de Ingenierías con un $20.42 \%$ y $16.72 \%$ respectivamente, le sigue la División de Ciencias Económico Administrativas con un $15.96 \%$, quién, de igual forma que las anteriores Divisiones, ha logrado reducir este indicador para ubicarse en un $12.95 \%$. En el cuarto lugar se encuentra la División de Ciencias Biológicas y de la Salud con un $13.33 \%$ y $10.41 \%$ actual. Para las otras Divisiones, la reducción del indicador no es tan significativa como en las anteriores, ya que en Ciencias Sociales pasó de $11.72 \%$ a un $11.04 \%$, mientras que en Humanidades que era de $8.73 \%$ se redujo a $8.17 \%$.

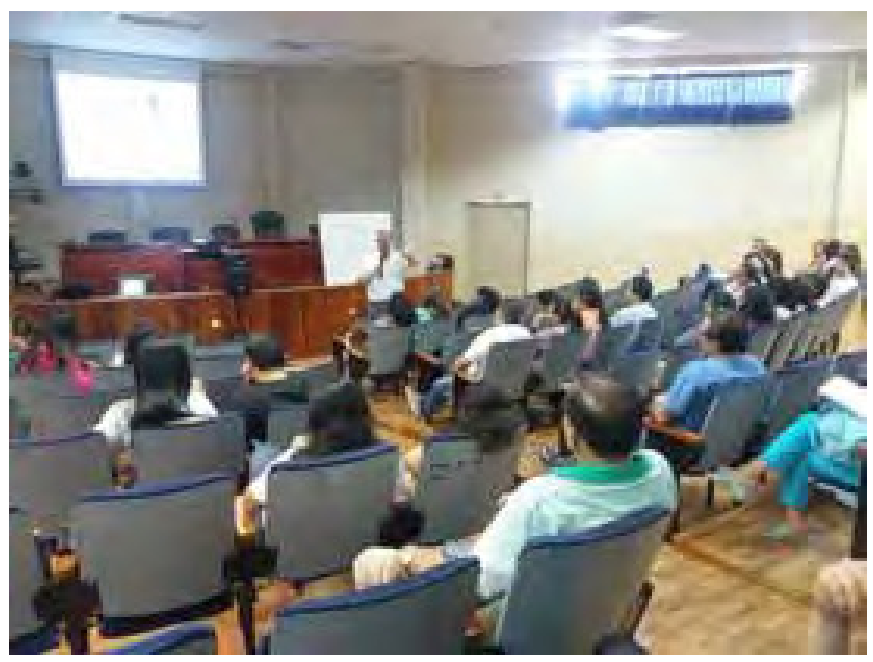

\section{Índice de reprobación por Licenciatura.}

A partir de la misma información proporcionada por la Dirección de Planeación del semestre 2004-1 al 2007-1 y del 2004-1 al 2016-2, y toda vez que se detectaron las dos Divisiones con mayor índice de reprobación, por programa educativo y en el mismo orden de idea de la secciones anteriores, los primeros lugares pertenecen a: Ingeniero Minero (33.12\% pasó a $22.46 \%)$, Ingeniería en Sistemas de Información (30.62\% a 20.63\%), Ciencias de la Computación (30.59\% a 28.62), Licenciatura en Matemáticas (27.60\% a 20.06), Ingeniero Agrónomo (24.53\% a $22.19 \%)$, Ingeniería en Tecnología Electrónica (22.53\% a 23.54\%), Licenciatura 
en Geología (22.06\% a $17.78 \%)$, Ingeniería Civil (21.19\% a 17.83), Ingeniería Química (20.10\% a $18.82 \%)$, Licenciatura en Física (19.57\% a $16.36 \%)$. Todos ellos muy por encima de la media institucional actual del $12.37 \%$, y con la característica de que sus planes de estudio contemplan asignaturas de Física y Matemáticas.

\section{Aprovechamiento por División}

En lo que respecta al rendimiento académico de los estudiantes, nuevamente las Divisiones de Ciencias Exactas y Naturales e Ingeniería ocupan los dos primeros lugares al poseer los más bajos promedios de calificación obtenida por sus estudiantes en el periodo comprendido del 2004-1 al 2016-2.

Así, la División que cuenta con el mejor aprovechamiento o promedio general de calificaciones es Humanidades y Bellas Artes con 79.74, Ciencias Sociales de 78.76, Ciencias Biológicas y de la Salud 77.78, las cuales se ubican por arriba de la media institucional (76.07). Las Divisiones que se encuentran por debajo de esta media promedio son: Ciencias Económico Administrativas de 74.80, Ingenierías 70.67 y la menos favorecida, Ciencias Exactas y Naturales con un promedio de aprovechamiento de los alumnos del 69.63

Por programa educativo, de las dos Divisiones menos favorecidas, ninguna de sus Licenciaturas sobrepasa el indicador institucional de 76.07, teniendo los alumnos de la Licenciatura en Física el mismo promedio institucional, seguido por Mecatrónica (76.06), Químico Biólogo Clínico (74.87), Materiales (73.39), Matemáticas (72.85), Químico en Alimentos (72.83), Ing. Química (72.44), Industrial (71.55), Geólogo (70.51), Químico Biólogo (70.25), Civil (69.78), Sistemas de Información (68.19), Electrónica (66.4), Minero (66.24) y en último lugar se tiene a la Licenciatura en Ciencias de la Computación (66.04).

Deserción en la División de Ciencias Exactas y Naturales.

Al ser la División de Ciencias Exactas y Naturales la menos favorecida de la Unidad regional Centro, se realizó un revisión documental de los alumnos que han ingresado desde el 2000 a alguna de sus cinco Licenciaturas. Para ello, se utilizó como referencia la base de datos proporcionada por la Dirección de Servicios Escolares y actualizada al semestre 2016-2.

En este periodo de 16 años, los Programas Educativos admitieron 3495 alumnos de los cuales 968 abandonaron sus estudios (46.15\%). La Licenciatura en Ciencias de la Computación tiene el mayor porcentaje de deserción (62.43\%), seguido por Matemáticas (54.46\%), Electrónica (52.03\%), Física (35.36\%) y Geología (33.39\%).

\section{6.- Reprobación por tercera ocasión de una misma asignatura.}

En estos 16 años, pero centrando la atención en los alumnos a quienes se les aplicó el reglamento escolar
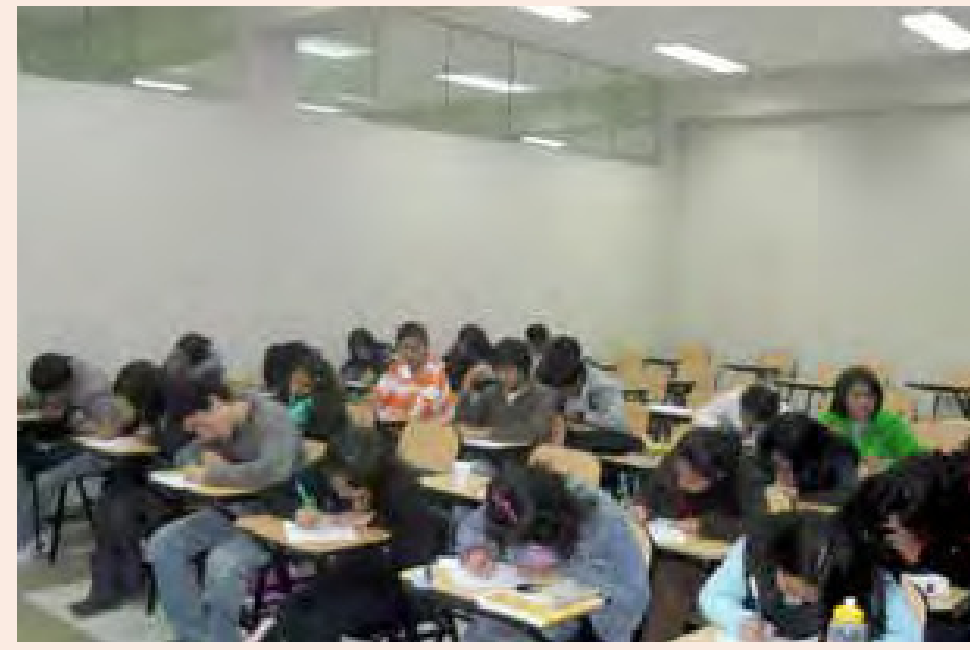

(Art. 64) y causaron suspensión temporal por reprobar una misma materia por tres ocasiones, los alumnos de Electrónica presentan el mayor porcentaje por este motivo (13.23\%), le sigue Computación (13.13\%) y por debajo de la media divisional $(9.35 \%)$, se encuentra Física $(7.46 \%)$, Geología (7.40\%), y Matemáticas (5.14\%), si bien es cierto que algunos alumnos solicitan su cuarta oportunidad mediante el examen extraordinario especial y al acreditarlo reanudan sus estudios, no es así para la gran mayoría que cayeron en esta situación.

\section{7.- Asignaturas más difíciles de acreditar.}

Siguiendo la misma dinámica de seleccionar la División y Programa Educativo menos favorecido, en el contexto de porcentaje de alumnos suspendidos temporalmente por reprobación en un periodo de 12 años que abarca desde el año 2000 al 2012, se tiene que las asignaturas que no pudieron acreditarse en tercera inscripción en la División de Ciencias Exactas, la Licenciatura en Tecnología Electrónica es la que tiene el mayor porcentaje en alumnos suspendidos debido a Cálculo Diferencial e Integral I, Mecánica I y Cálculo Diferencial e Integral II, en las cuales 45 de un total de 541 alumnos, no pudieron acreditarlas, es decir, estas tres materias originaron un $8.31 \%$ de alumnos suspendidos por reglamento escolar. Considerando que los alumnos que ingresan a la Licenciatura en Física, lo hacen con la más firme convicción, a la par que adquirieron en su formación previa bases firmes en el área de físicomatemáticas, al revisar las trayectorias escolares de ocho generaciones de alumnos (2009 a 2016), se encontró que las principales asignaturas que causaron la aplicación normativa de suspensión temporal fueron: Mecánica I (6 alumnos), Calculo Diferencial e Integral I, y II, Álgebra lineal (5 alumnos c/u), Algebra superior, Geometría Analítica y Electromagnetismo ( 3 alumnos $\mathrm{c} / \mathrm{u}$ ) es decir, asignaturas de los primeros semestres del área de ciencias básicas (Física y de Matemáticas). Si esto ocurre en esta Licenciatura, ¿qué se puede esperar de otras Licenciaturas que contienen estas mismas asignaturas? Para dar una idea de tal problemática, los resultados de un estudio de trayectorias 
escolares de los alumnos de la División de Ingenierías que ingresaron del 2000 al 2012, muestra que ingresaron 7767 alumnos distribuidos en siete licenciaturas. De ellos, causaron suspensión temporal 389 alumnos (5\%), donde 110 alumnos eran de Civil, 151 de Industrial, 18 de Minas, 8 de Mecatrónica, 29 de Ing. Química y 73 de Sistemas de Información. Las asignaturas que no pudieron acreditar los alumnos en tercera oportunidad fueron: Calculo Diferencial e Integral II (63 alumnos), Cálculo Diferencial e Integral I (51 alumnos), NTIC (41 alumnos), Álgebra (34 alumnos), Geometría Analítica (26 alumnos), Calculo Diferencial e Integral III (22 alumnos), Ecuaciones Diferenciales I (18 alumnos), Física I (16 alumnos), Probabilidad y Estadística (12 alumnos), Fluidos y electromagnetismo (4), Física II (3 alumnos) y Física III ( 2 alumnos). El mismo estudio indica que se debe reforzar el apoyo a estudiantes de Sistemas de Información, Civil e Industrial en Calculo I y II, a los alumnos de Ing. Química, Sistemas de Información e Industrial en Calculo III, Álgebra a los alumnos de de Minas, Industrial y Civil, por mencionar algunas asignaturas y programas educativos como ejemplo.

\section{DESARROLLO.}

En su inicio en el semestre 2007-2, por los índices de reprobación, bajo aprovechamiento y rezago en asignaturas que imparte el Departamento de Física [13], que conllevan al desgranamiento escolar, deserción y consecuentemente a la baja eficiencia de egreso en los programas educativos de las Divisiones a las cuales se les brinda servicio, el programa de asesoría de pares fue concebido para atender dicha problemática.

A partir de la convivencia e interacción social generada entre estudiantes de nuevo ingreso y los estudiantes de Física de los últimos semestres en asignaturas de Física, surge la solicitud de ayuda en asignaturas de matemáticas, incorporándose asesorías de Cálculo Diferencial e Integral I, II y III, Álgebra, Geometría Analítica, Probabilidad y Estadística, Análisis Numérico y Ecuaciones Diferenciales.

Con el devenir del tiempo, el programa de asesoría de pares empieza a crecer y es solicitado por alumnos de la Licenciatura en Matemáticas para realizar el Servicio Social

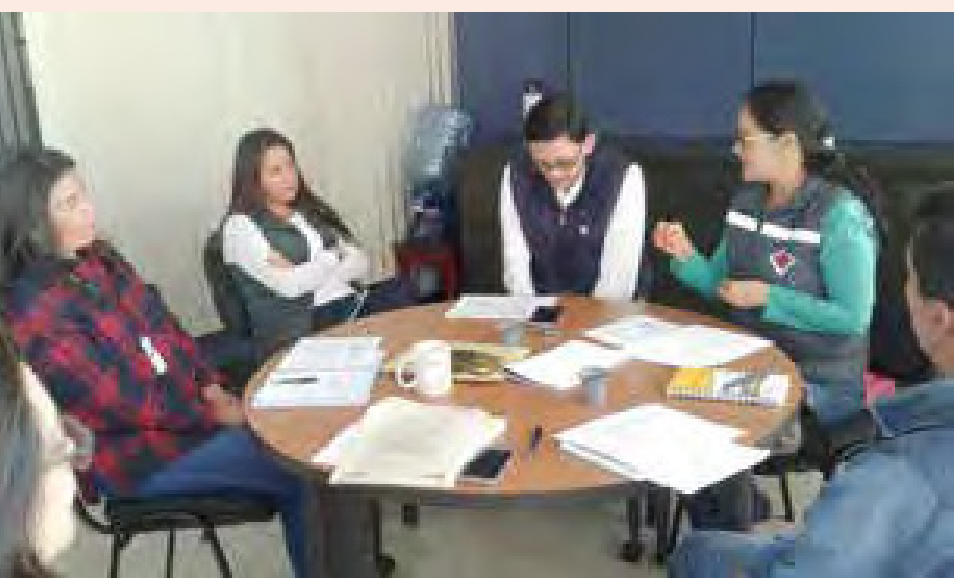

Universitario. A la fecha, no solo se atienden asignaturas de Física y Matemáticas, se han incorporado asesores pares en la modalidad de prestadores de Servicio Social provenientes de la División de Ingenierías, quienes imparten asignaturas propias de esos programas educativos.

\section{Fuentes de Financiamiento.}

El proyecto, circunscrito al Proyecto de Sala DidácticaAsesorias del Departamento de Física, desde su inicio a sido apoyado por: Jefatura del Departamento, Dirección de la DCEN, Dirección de Servicios Estudiantiles, Dirección de Servicios Escolares, Dirección de Investigación y Posgrado, Secretaría General Académica, así como por órganos personales y colegiados, otorgándose recursos económicos para adquisición de materiales y equipo de uso diario (marcadores, hojas, toners, impresión de trípticos de difusión, uso de copiadora, agua, impresora, equipo de computo, servicio de mantenimiento a espacios físicos, etc), así como el otorgamiento de becas ayudantía y recursos económicos para presentación de ponencias en eventos académicos regionales y nacionales.

\section{Proceso de selección de alumnos asesores pares. i) Prestadores de Servicio Social}

En todos los semestres se participa en convocatoria publicada por la Dirección de Servicios Estudiantiles solicitando prestadores de Servicio Social, para lo cual el responsable del proyecto registra el proyecto de asesores pares. En él se especifican las Licenciaturas de los prestadores solicitados, así como las características especiales y cualidades personales requeridas, entre las que se pueden mencionar: compromiso, responsabilidad e interés por ayudar a alumnos de los primeros semestres y compañeros, conocer lo que es y saber trabajar en equipo, facilidad para entablar conversación con pares, comunicación clara, ser crítico y utilizar su juicio de forma adecuada en relación con diferentes personalidades y situaciones que se le presenten, conocimiento de la Institución tanto en aspectos normativos como de ubicación de espacios físicos, así como de programas y servicios de apoyo a estudiantes, ser buen estudiante con conocimientos sólidos de la(s) asignatura(s) que va a asesorar y donde él sienta que es su mayor fortaleza, disposición a capacitarse para ejercer la función de asesor par. Entre las funciones que desarrollará se encuentran: difundir el programa de asesoría entre pares, proporcionar información de utilidad a sus asesorados, compartir sus experiencias y ayudarles a su adaptación al entorno resolviendo dudas tanto de información institucional como conceptual de la asignatura asesorada. En el proceso de selección, los aspirantes a realizar el Servicio Social como asesor par, son entrevistados por el responsable del proyecto de Servicio Social, quién constatará mediante entrevista estructurada que se reúnan la mayoría de las características y cualidades descritas anteriormente y verifica mediante revisión de Kardex su desempeño 
estudiantil. En todos los casos, se les solicita la anuencia del Coordinador de su Licenciatura y una carta de recomendación de su profesor-tutor o del profesor que le impartió la asignatura que pretende asesorar.

\section{ii) Ayudante de profesor}

Se aplica en todos los semestres por parte del responsable y/o colaboradores del proyecto de asesoría de pares en la convocatoria de becas ayudantía emitida por el H. Consejo de la División de Ciencias Exactas y Naturales. De aprobarse, el profesor beneficiado propone, atendiendo a la normatividad vigente, al alumno que a su juicio reúna los requisitos para desempeñarse como asesor par o bien, podrá proponer a algún alumno que haya realizado su Servicio Social como asesor par y que haya sobresalido en el número de alumnos atendidos durante su Servicio.

\section{iii) Alumnos voluntarios}

Son aquellos alumnos que cumpliendo algunas características y cualidades descritas anteriormente, así como haber cursado alguna asignatura de forma sobresaliente, tengan la vocación de servir y apoyar a pares de alumnos o a sus mismos compañeros que no acreditaron la asignatura en la que sobresalieron.

\section{Proceso de capacitación.}

A los alumnos seleccionados se les cita a inicio de semestre, por parte del responsable y colaboradores del proyecto, a una reunión grupal en la Sala DidácticaAsesorías, es de carácter informativo y capacitación para reafirmar sus conocimientos sobre la organización, infraestructura, normatividad y servicios institucionales, con participación voluntaria de alumnos que se han desempeñado como asesores paresa efectos de que se transmitan experiencias.

\section{Proceso de difusión.}

Al finalizar la reunión de capacitación, se elabora el programa de asesoría de pares del semestre correspondiente, que contiene los nombres de los asesores pares, días, horarios y asignaturas que asesorarán. Se edita y se plasma en un tríptico con un tiraje que va desde 500 a 1000 trípticos. Se publica durante las dos primeras semanas del periodo lectivo mediante la distribución en aulas y pegado en sitios visibles y de alta afluencia de estudiantes. Se hace entrega de varios ejemplares a los Coordinadores de Programa Educativo para su conocimiento y difusión, en cuyos planes de estudio se contemplen las asignaturas que se asesorarán. De igual forma, el tríptico se pone a disposición en formato electrónico vía Web y redes sociales. En la medida de las posibilidades, se contacta a profesores que imparten las asignaturas ofertadas para que canalicen al proyecto de asesoría de pares a los estudiantes que ellos estimen se encuentran en riesgo escolar.

Se pretende establecer contacto permanente con Coordinadores Divisionales de Tutorías de las Divisiones

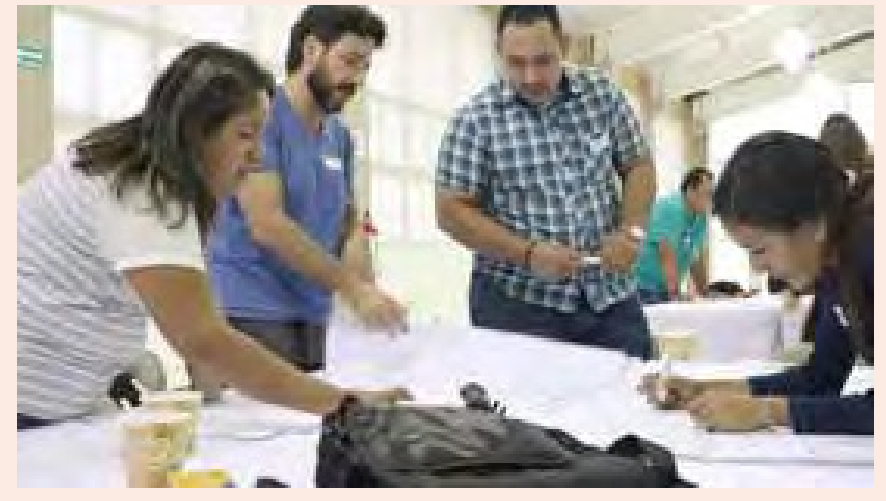

de Ingenierías, Ciencias Exactas y Naturales, Químico Biológicas y de la Salud, así como de Humanidades y Bellas Artes, para ofrecer y brindar apoyo a los alumnos que el Programa de Mejoramiento de Trayectorias Escolares (PROMETE) auspiciado por la subdirección de Tutorías de la Dirección de Servicios Estudiantiles, consideren que alumnos deben ser canalizados a asesorías en asignaturas tanto de Física como de Matemáticas de los primeros semestres, al ser alumnos detectados como vulnerables o encontrarse en riesgo escolar por reprobación y que pueden causar suspensión temporal por aplicación del reglamento escolar.

En el mismo contexto Divisional, se contempla compartir experiencias con los responsables de Proyectos de Asesoría de Pares de otros Departamentos o Programas Educativos para conjuntar esfuerzos encaminados a apoyar a estudiantes y mejorar el presente proyecto, iniciando con su socialización y conocimiento como lo es el caso del presente escrito, que permita enriquecerlo de experiencias. Así mismo, se incluirá y compartirá con los responsables de las otras Unidades Regionales de la Universidad de Sonora a través de la Subdirección de Tutorías de la Dirección de Servicios Estudiantiles.

\section{Proceso de seguimiento y desarrollo.}

Cada bisemana se sostiene una reunión con los alumnos asesores pares para constatar avances o retrocesos del proyecto para su redimensión o retroalimentación. Se revisa la bitácora donde se registran los alumnos constatando su afluencia así como las asignaturas, días y horarios más demandados y tomar acciones para su reforzamiento.

Por parte del responsable y colaboradores del proyecto (profesores), además de las actividades descritas en la selección de alumnos y las inherentes a la normatividad respectiva, tienen la obligación de brindar atención inmediata a sus alumnos asesores pares o ayudantes de profesor, cuando a ellos se les presente alguna dificultad o duda en algún tema o problema relacionado con su especialidad.

Los asesores pares, en cumplimiento con responsabilidad del compromiso adquirido, además 
de brindar orientación institucional, participar en la difusión del programa, atender dudas conceptuales o planteamiento y solución de problemas en la asignatura asesorada, atienden las actividades descritas en los proyectos de Servicio Social o Beca Ayudantía, así como vigilar el registro diario en bitácora de los alumnos que atiende, recopilando y transcribiendo de ellos los problemas o tareas asignadas por los profesores, esto con la finalidad de formar un banco de reactivos por materia, el cual se heredará a futuras generaciones de alumnos asesores pares. Adicionalmente, tendrán que cumplir con las obligaciones inherentes del Servicio Social o Becas Ayudantía, elaborando y entregando con oportunidad los informes correspondientes.

\section{Proceso de evaluación.}

Esta fase está en proceso de desarrollo, en la cual, de forma aleatoria y anónima, los asesores pares solicitarán a alumnos asesorados llenar una encuesta de opinión y satisfacción del apoyo que se les brindó, la cual se depositará en urna sellada que será abierta al finalizar el periodo escolar, realizando conjuntamente con asesores pares y colaboradores del proyecto el informe respectivo. Al finalizar el periodo lectivo, se procederá a capturar los registros en bitácora en hoja de Excel, determinando entre otros indicadores la frecuencia de asistencia de alumnos por asignatura, por Licenciatura y por División. Mediante autoevaluación practicada por el responsable y colaboradores, se determinará el impacto del Proyecto de Asesores Pares en indicadores o metas institucionales tales como:

A) Número de asesorías y alumnos atendidos en asesoría de pares.

B) Tasa de rendimiento (porcentaje de alumnos que aprobaron las materias asesoradas).

C) Promedio de calificaciones por materia (aprovechamiento)

D) Índice de reprobación por materia.

Los cuales se reportarán en forma global de resultados en formato de tabla y/o gráfico por División y por Licenciatura. Se contrastarán dichos resultados con los obtenidos en el semestre previo por el mismo proyecto de asesores pares, así como con los publicados en el semestre anterior por la Dirección de Planeación en su página electrónica.

Para la evaluación por parte de órganos personales y colegiados del Proyecto de Asesoría de Pares en término de los indicadores y metas institucionales correspondientes que se encuentran enmarcados en el Plan de Desarrollo Institucional, se enviará una copia fotostática de los registros en bitácora para que dicha información sea procesada y analizada en término de los expedientes de los alumnos que acudieron a solicitar ayuda.

\section{Requisitos para la autoevaluación.}

Para cumplir con el compromiso de la autoevaluación e informar sobre el cumplimiento de metas, es requisito indispensable contar con acceso a la revisión en línea de Kardex de los alumnos que asistieron a asesorarse, la cual se hará con fines exclusivamente académicos, con apego irrestricto al código de conducta y código de ética Institucional, con profesionalismo y bajo la más estrictas reservas de confidencialidad y secrecía de la información consultada.

\section{BIBLIOGRAFÍA}

[01] ANUIES (2000). Programas Institucionales de Tutorías. Una propuesta de la ANUIES para su organización y funcionamiento en las instituciones de educación superior. Extraído el 2 de mayo de 2008 desde: http://www.ANUIES. $\mathrm{mx} /$

[02] UNESCO (2004). Las tecnologías de la información y la comunicación en la formación docente: guía de planificación. Organización de las Naciones Unidas para la Educación, la Ciencia y la Cultura

[03] Amaya Fragoso, E., Angulo Amarillas, S.E y González Barceló, F.J. (2011). Programa académico de asesorías en pares para el Departamento de Medicina y Ciencias de la Salud. III Foro Institucional de Tutorías: Experiencias y reflexiones para el seguimiento y mejora de trayectorias escolares en la Universidad de Sonora. Octubre, Hermosillo, Sonora. Consultado el 20 de diciembre del 2016 en http://www.dise. uson.mx/TUTORIAS/IIIFOROTUTORIAS/40.PDF

[04] Tinto, V. (1989). Definir la deserción: una cuestión de perspectiva. Revista de la Educación Superior. Vol. XVIII (3), número 71, julio-septiembre de 1989.

[05] Jiménez García, F. I., Torres Bezaury, R. (n.d.). Propuesta para la formación de asesores. Extraído el 2 de mayo de 2008 desde: http://www.te.ipn.mx/1erencuentrotutoria/ archivos/1C08.doc

[06] Castro Ricalde, D. y López Arriaga, A. (2006). El papel del tutor adjunto, como complemento académico de la actividad tutorial. Trabajo presentado en el Segundo Encuentro Nacional de Tutoría, Octubre, Monterrey N.L. México.

[07] Alamilla Acevedo, A. N. y Puga Sandoval, C. V. (2006). Metodología del Programa de Tutoría entre Iguales en la Facultad de Educación Unidad Tizimín. Trabajo presentado en el Segundo Encuentro Nacional de Tutoría, Octubre, Monterrey N.L. México.

[08] Burgos Vidal, W. E., Couoh Tuz, L.R. y Quintana Alcalá, M. M. A. (2006). El proceso de tutoría entre iguales en el Nivel Superior: Programa implementado en la universidad Autónoma de Yucatán. Facultad de Educación-Unidad Tizimín. Trabajo presentado en el Segundo Encuentro Nacional de Tutoría, Octubre, Monterrey N.L. México.

[09] Cantú Martínez, L. M., Siller Botti, M.G. y García Treviño, V. (2006). La Tutoría de Pares, como estrategia para atender la diversidad de la población estudiantil de la UDEM. Trabajo presentado en el Segundo Encuentro Nacional de Tutoría, Octubre, Monterrey N.L. México.

[10] [11] [12] Pacheco Arriquives, M. A., Burgos Flores, B. (2007, 2008, 2010). Estudio de Deserción. Colección documentos de Investigación Educativa. Universidad de Sonora. México. Recuperado de http://www.planeacion.uson.mx/ estudiantes.htm

[13] Cruz Encinas, I., Armenta Aguilar, F. (2005). Estudio de Trayectorias Escolares en asignaturas de Física: Generación 1999. Colección Documentos de Investigación Educativa. Universidad de Sonora. 